Int. J. Morphol.,

35(2):751-755, 2017

\title{
Los Orígenes de la Sociedad de Anatomía Normal y Patológica de Chile
}

\author{
The Origins of the Society of Normal and Pathological Anatomy of Chile
}

\author{
Alberto Rodríguez Torres ${ }^{1}$ \& Julio Cárdenas Valenzuela ${ }^{2}$
}

RODRÍGUEZ, T. A. \& CÁRDENAS, V. J. Los orígenes de la Sociedad de Anatomía Normal y Patológica de Chile. Int. J. Morphol., 35(2):751-755, 2017.

RESUMEN: Desde los inicios, anatomistas y patólogos se unen en el año 1938 creando la Sociedad de Anatomía Normal y Patológica, con el fin de estimular sus investigaciones, buscar relacionarse tempranamente con morfólogos del resto de América y potenciar sus publicaciones en los Archivos Chilenos de Morfología. Es así como morfólogos chilenos provenientes de las principales Universidades del país participan por primera vez en Congresos y Jornadas tanto nacionales como internacionales. Quizás el trabajo en común con los cadáveres fue un punto fundamental en sus inicios, más el avance de los conocimientos en cada una de estas disciplinas y diferencias políticas posteriores serían dos hechos que explicarían que se separasen posteriormente en el año 1970. Sería por tanto, el primer esfuerzo de reunirse en torno a una Sociedad de Morfólogos en Chile, la base para la futura Sociedad Chilena de Anatomía.

PALABRAS ClAVE: Sociedad de Anatomía Normal y Patológica de Chile; Historia.

\section{Historia de un comienzo}

Después de 100 años de iniciarse los estudios médicos y anatómicos en Chile incorporados a una Universidad (Ferrer, 1904), el 27 de Diciembre de 1933 Gustavo Jirón Latapiat, académico de Anatomía de la Universidad de Chile crea los Archivos Chilenos de Morfología, siendo su primer Director. La citada publicación permite cubrir la necesidad de tener un órgano escrito, en el cual los anatomistas y académicos relacionados con el área morfológica tuviesen un espacio de creación, divulgación y comunicación con morfólogos extranjeros.

En el Tomo I de dichos archivos se destaca un concienzudo artículo acerca de la Historia de la Anatomía en Chile hasta el siglo XIX, escrito por el Dr. Néstor Flores W. (Flores, 1933) Ayudante de Anatomía; un artículo “Anatomía del recién nacido «La Cavidad Abdómino - Pelviana», estudio efectuado por la Dra. Erna Mahn H., memorista de Anatomía, con disecciones en un universo de 800 fetos de término y una breve monografía «Sobre cisuras pulmonares» escrita por el Prof. Gustavo Jirón, que fue mundialmente conocida. Todos los autores citados pertenecían a la Escuela de Medicina de la Universidad de Chile. (Fig. 1).

\section{La Sociedad de Anatomía Normal y Patológica}

Inicio de la Sociedad. Progresivamente fue surgiendo la necesidad de aglutinar en una entidad formal, éstas y otras iniciativas de morfólogos docentes e investigadores dispersos en los ámbitos universitario y hospitalario. Las gestiones culminan en la fundación de la Sociedad de Anatomía Normal y Patológica de Chile. Ésta sesiona por primera vez el 12 de Agosto de 1938, en dependencias de la Sociedad Médica de Chile (Archivos Chilenos de Morfología, 1940). Llamativa es la visión de convocatoria para esta iniciativa, pues precede a la Sociedad Mexicana de Anatomía, que se fundaría recién en 1957, y a la Sociedad Rioplatense de Anatomía en 1964. Fig. 2.

Esta sesión constitutiva fue presidida por el Dr. Gustavo Jirón, Académico y Profesor de Anatomía en el Instituto de Anatomía de la Universidad de Chile.

Los socios fundadores fueron 35 , y sus nombres se detallan a continuación: Albertz Alberto, Aliaga Claudio, Apablaza Hernán, Barrientos Juvenal, Benavente Ricardo Braemer Hardy, Brieba Isaac, Brink Guillermo, Calderón Eduardo, Carvajal Oscar, Croizet Emilio, Davanzo Ramón, Escobar Adolfo, Espíldora Cristóbal, Fernández Walter, Flores Néstor, Guzmán Alberto, Jirón Gustavo, Henckel Carlos, Herzog Ernesto, López Moisés, Mena Ismael, Muñoz Basilio, Muñoz Nicolás, Peña Ernestina, Rencoret Rodolfo, Rodríguez Héctor, Rodríguez Ramón, Sandoval Luis, Schneider Carlos, Sievers Hugo, Solervicens Enrique, Wainstein Abraham, Wilhelm Ottmar y Zúñiga Eduardo. Fig. 3.

\footnotetext{
${ }^{1}$ Médico Cirujano, prof. Titular ICBM, Facultad de Medicina, Universidad de Chile, Santiago, Chile.

${ }^{2}$ Médico Cirujano, internista, prof. Asistente ICBM, Facultad de Medicina Universidad de Chile, Santiago, Chile.

Programa Anatomia y Biología del desarrollo, ICBM, Facultad de Medicina, Universidad de Chile, Santiago, Chile.
} 
Sociedad de Anatomía Normal y Patológica de Chile. Acta de sesión inaugural de la Sociedad de Anatomía Normal y Patológica de Chile. Archivos Chilenos de Morfología. Vol.3. Biblioteca Instituto de Anatomía Universidad de Chile.

Las actividades de la Sociedad se realizaban a través de reuniones, con presentación de trabajos vinculados a la Morfología tanto Normal como Patológica, y a la realización de congresos nacionales, sin una periodicidad pre-establecida. Participaban en estas actividades morfólogos macro y microscopistas, humanos y veterinarios, con estudios y aplicación de temas vinculados a organismos normales y patológicos.

Como requisito de ingreso para constituirse como miembro activo de la Sociedad, se precisaba la presentación de un trabajo original de índole morfológica, fuese éste normal o patológico, debiendo haberse aprobado previamente sus antecedentes por miembros calificados como

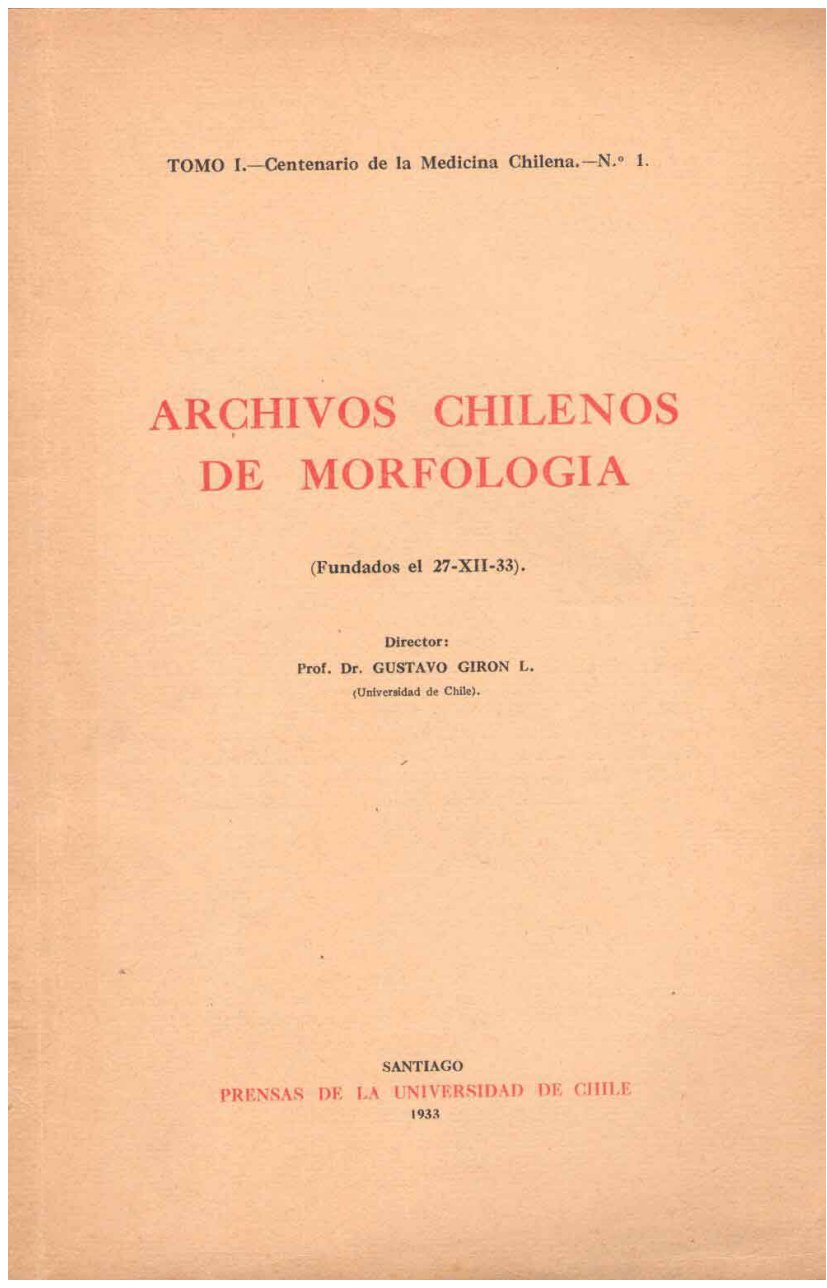

Fig. 1. Portada del primer número de los Archivos Chilenos de Morfología. informantes, para luego exponer en forma pública el trabajo en cuestión. Fig. 4.

Los centros de actividad eran para la Morfología Normal: la Universidad de Chile con sus Facultades de Medicina y Odontología en Santiago y su sede en Valparaíso, la Universidad Católica de Chile y la Universidad de Concepción. Muy posterior a estos centros formadores de la Morfología normal, aparece y muy incipiente, la Universidad Austral de Valdivia, en 1964.

Para la Anatomía Patológica los centros involucrados en su enseñanza eran las Universidades antes citadas además de los Servicios de Anatomía Patológica de hospitales de Santiago (principalmente los del Salvador, San Borja y San Juan de Dios) y los Servicios correspondientes en Valparaíso, Concepción y, posteriormente, Valdivia.

Desde la fundación de la Sociedad en 1938, los Archivos Chilenos de Morfología pasaron a ser el órgano oficial de la Sociedad de Anatomía Normal y Patológica. Estos Archivos surgieron con el fin de mejorar el estudio de las Ciencias Morfológicas, "relegadas a segundo término en estos pueblos jóvenes cuyas universidades estimulan de preferencia cuanto tiene relación con la aplicación práctica de las profesiones liberales" (Jirón, 1946).

\section{SOCIEDAD DE ANATOMIA NORMAL Y PATOLOGICA DE CHILE \\ SOCIOS FUNDADORES}

Gustavo Girón L., Héctor Rodríguez, Néstor Flores W., Ramón Rodríguez T., Luis Sandoval, Nicolás Muñoz H., Ernesto Herzog, Carlos Henckel, Ottmar Wilhelm, Enrique Solervicens, Carlos Oliver Schneider, Hernán Apablaza, Hardy Braemer, Oscar Carvajal D., Ramón Davanzo, Moisés López, Adolfo Escobar, Abraham Wainstein, Basilio Muñoz Pal, Walter Fernández B., Emilio Croizet, Carlos Aliaga, Guillermo Brink, Cristóbal Espíldora L., Ismael Mena, Ricardo Benavente G., Rodolfo Rencoret, Hugo K. Sievers W., Eduardo Zúñiga R., Ernestina Peña, Alberto Albertz, Eduardo Calderón Paul, Juvenal Barrientos R. Isaac Brieba, Alberto Guzmán.

$$
\text { Socios HoNorarios }
$$

Profesor Dr. Juan Noé; Profesor Dr. David Benavente; Profesor Dr. Carlos E. Porter; Profesor Dr. Pedro Belou, Honorario extranjero Buenos Aires; Profesor Dr. Angel H. Roffo, Honorario extranjero Buenos Aires.

\section{SESION INAUGURAL}

Primera Sesión

En Santiago, a 12 de Agosto de 1938 se constituyeron en el local de la Sociedad Médica de Santiago a las 19 horas y 30 minutos, los señores :

Gustavo Girón L., Profesor de Anatomía de la Escuela de Medicina de la Universidad de Chile; Enrique Solervicens, Profesor de Anatomía de la Universidad dê Concepción: Carlos Henckel, Frofesor de Anatomía Comparada Embriología e Histología de la Universilad de Concepcion, Héctor Rodúcón Ańtomo Patólogo del Hospital San Bón, Hugo H Sievers W. Decano de la Facula Hugo H. Sievers W., Decano de la Facultad de Medicina Veterinaria, Profesor de Patología; Abraham Wainstein, Jefe de trabajos prácticos de Anatomía en la Escuela Dental, de la Universidad de Chile; Ernesto

Fig. 2. Primera página de la Constitución de la Sociedad de Anatomía Normal y Patológica de Chile. 

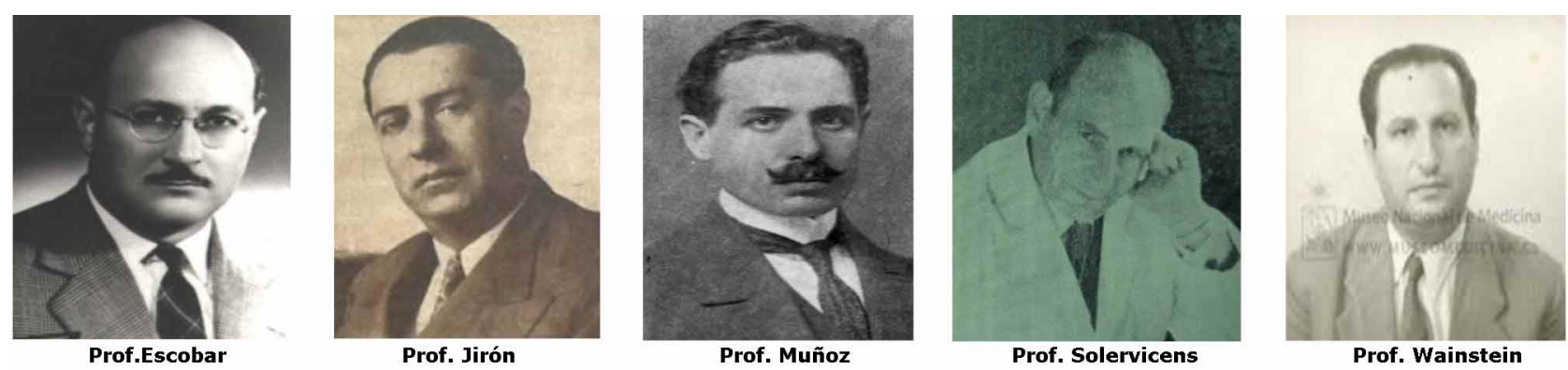

Fig. 3. Profesores de Anatomía, participantes de la creación de la Sociedad de Anatomía Normal y Patológica. Gentileza Museo de la Medicina, Facultad de Medicina, U. de Chile.

En la Sociedad de Anatomía Normal y Patológica de Chile se reunieron los cultores de Anatomía, Histología, Anatomía Patológica, Antropología Física, Humana y Veterinaria. Si bien es cierto que se reconoce el gran y valioso aporte europeo en estos conocimientos, también se precisó la necesidad de tener este centro donde poder reunir los conocimientos que se estaban gestando en nuestro país. Se establece en sus primeros estatutos la necesidad de interrelacionarse con instituciones similares de otros países especialmente de este continente, muchas de ellas desconocidas, estimulando las investigaciones en conjunto, agregando las modalidades morfológicas étnicas de nuestros pueblos, estrechando el nexo espiritual que debe unir a nuestros hombres de estudio., "comprometiéndose a publicar todos los trabajos de la Sociedad, con los que se honrarán estas páginas.” (Archivos Chilenos de Morfología, 1940).

Se recuerda como el hecho de mayor connotación de la Sociedad de Anatomía Normal y Patológica, la realización del Congreso Nacional de Ciencias Morfológicas el que tuvo carácter internacional y fue inaugurado el 17 de Noviembre del año 1942, con motivo del Centenario de la Universidad de Chile. Se puede considerar como el Primer Congreso Panamericano de Anatomía realizado en nuestro país

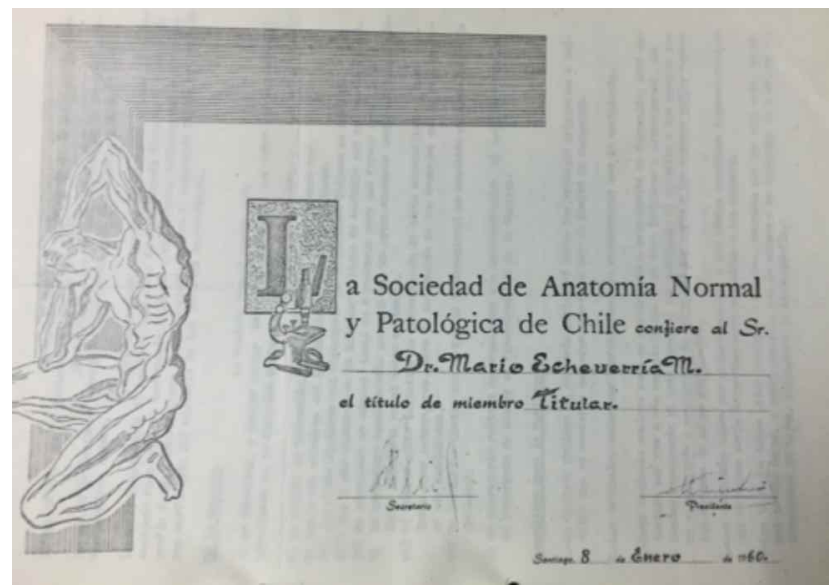

Fig. 4. Certificado de miembro Titular de la Sociedad, archivos de reuniones de Directorio, 1960. dado la concurrencia de morfólogos de Argentina, Brasil, Ecuador, Paraguay, Perú y Uruguay anticipándose a la entidad que después se fundaría como Asociación Panamericana de Anatomía, en la Ciudad de México, en 1966. En esta ocasión y junto con numerosos trabajos científicos en que destacaron los vinculados a la antropología física, se esbozaron líneas de acción conjunta de índole científica, en que se considerarían las variaciones regionales en el continente americano; éstas se difundirían en Latinoamérica a través de una editorial que imprimiera y diera a conocer los textos que se gestaran en esta acción colectiva.

Así, Gustavo Jirón expresó: "La Anatomía del Hombre blanco no es la anatomía de la humanidad entera", como había señalado años antes el Profesor Loth de Varsovia. (Jirón, 1939). Presenta una tesis: "Algunas consideraciones sobre la anatomía descriptiva y topográfica del bazo", uno de los puntos de partida para iniciar los trabajos anatómicos de la Anatomía chilena. Solervicens, en 1942, puso en relieve la diferencia observada en el sistema simpático abdominal, sobre el plexo solar ("masa solar") (Solervicens, 1946), Henckel y Skews estudiaron en 1953, el peso del bazo, corazón, hígado y pulmones; Moisés López lo hace en relación al drenaje de los vasos linfáticos, lo que Harnecker más adelante describiría con mayor exactitud, destacando sus diferencias en relación a la anatomía tradicional anglosajona. Es más, a partir de estos trabajos, los anatomistas del Cono Sur inician también sus estudios anatómicos regionales. Incluso H. Rouvière haría mención de Jirón sobre diferencias descritas en cisuras pulmonares y el lobo ácigos.

Gustavo Jirón propugnaba la creación de un texto Anatómico Latinoamericano, de lo que estaban de acuerdo los Profesores Ricardo Palma de Perú, Sosa de Uruguay y en Argentina Humberto Fracassi y Bianchi, entre otros, proponiendo repartirse los capítulos entre los países. Se debería poner énfasis en lo práctico de la Anatomía: « si voy a hablar del radio, me referiré a su fractura; si hablo de venas, hablaré de várices, dando un sentido a los conocimientos anatómicos, no olvidándonos que formaremos Médicos Cirujanos» decían estos últimos. 
Igualmente, Jirón propugnaba tener una mejor comunicación de los trabajos de investigación latinoamericanos, pues consideraba una incongruencia que "las relaciones científicas son más frecuentes con quienes se dedican al problema en Europa y a veces más allá, con la producción científica de investigadores asiáticos, en tanto que los trabajos de los amigos cercanos, del otro lado de la cordillera, de los límites de nuestra frontera, de nuestros hermanos de destino y de trabajo común, pasan inadvertidos para nosotros, para nuestros centros de estudio, para nuestros alumnos" (Archivos Chilenos de Morfología, 1942).

Desde la fundación de la Sociedad de Anatomía Normal y Patológica de Chile se efectuaron sesiones de presentación de trabajos sobre variados temas morfológicos sin una secuencia determinada, en jornadas programadas que incluían la Morfología normal y patológica, humana y animal. Aparte del ya mencionado Congreso Nacional efectuado el año 1942, destacaron, una Jornada Nacional efectuada en dependencias de la Biblioteca Nacional en Santiago y el $\mathrm{X}^{\circ}$ Congreso Nacional realizado en Noviembre de 1965, que fue presidido por el Prof. Dr. Carlos Medina, en la sede Playa Ancha - Valparaíso de la Facultad de Medicina de la Universidad de Chile (Fig. 5).

Un período de mucha actividad de la Sociedad fue el vivido en los años 1964 y 1965, bajo la presidencia del Prof. Dr. Abraham Wainstein S. Profesor de la Facultad de Odontología de la Universidad de Chile, quien además, desarrollaba su labor en el Instituto de Anatomía de la misma Uni- versidad. En ese periodo se promueve activamente el ingreso de docentes jóvenes que, posteriormente tendrían una amplia participación en la gestación y primeros años de existencia de la futura Sociedad Chilena de Anatomía.

Cabe destacar, promovido por la presidencia de la Sociedad, el ingreso en este período de profesionales jóvenes que se desempeñaban como ayudantes de Anatomía: los Médicos Doctores Evaristo Cancino Águila, Jorge Micheli C. y Alberto Rodríguez Torres, y los Odontólogos Doctores Humberto Guiraldes del Canto, Hugo Hernández Parada y Patricio Quezada Pacheco quienes cumplieron posteriormente un papel destacado en la transición a la futura Sociedad. Chilena de Anatomía (Fig. 6).

\section{Término de la Sociedad}

Durante algún tiempo y en forma irregular, se efectuaron jornadas citadas por el Directorio correspondiente, en las que ya era posible evidenciar un hecho sintomático que repercutiría a futuro para la estabilidad y mantención de la Sociedad: cuando citaban los patólogos a las jornadas, no asistían los anatomistas y cuando citaban éstos, aquellos tampoco lo hacían. Esto se explicaba probablemente, en virtud del interés disímil de los temas que se trataban.

Asímismo, se debe considerar que los centros de mayor actividad estaban en Santiago y Concepción, distantes $500 \mathrm{~km}$, lo que dificultaba el desplazamiento de socios, algunos de ellos de edad avanzada.

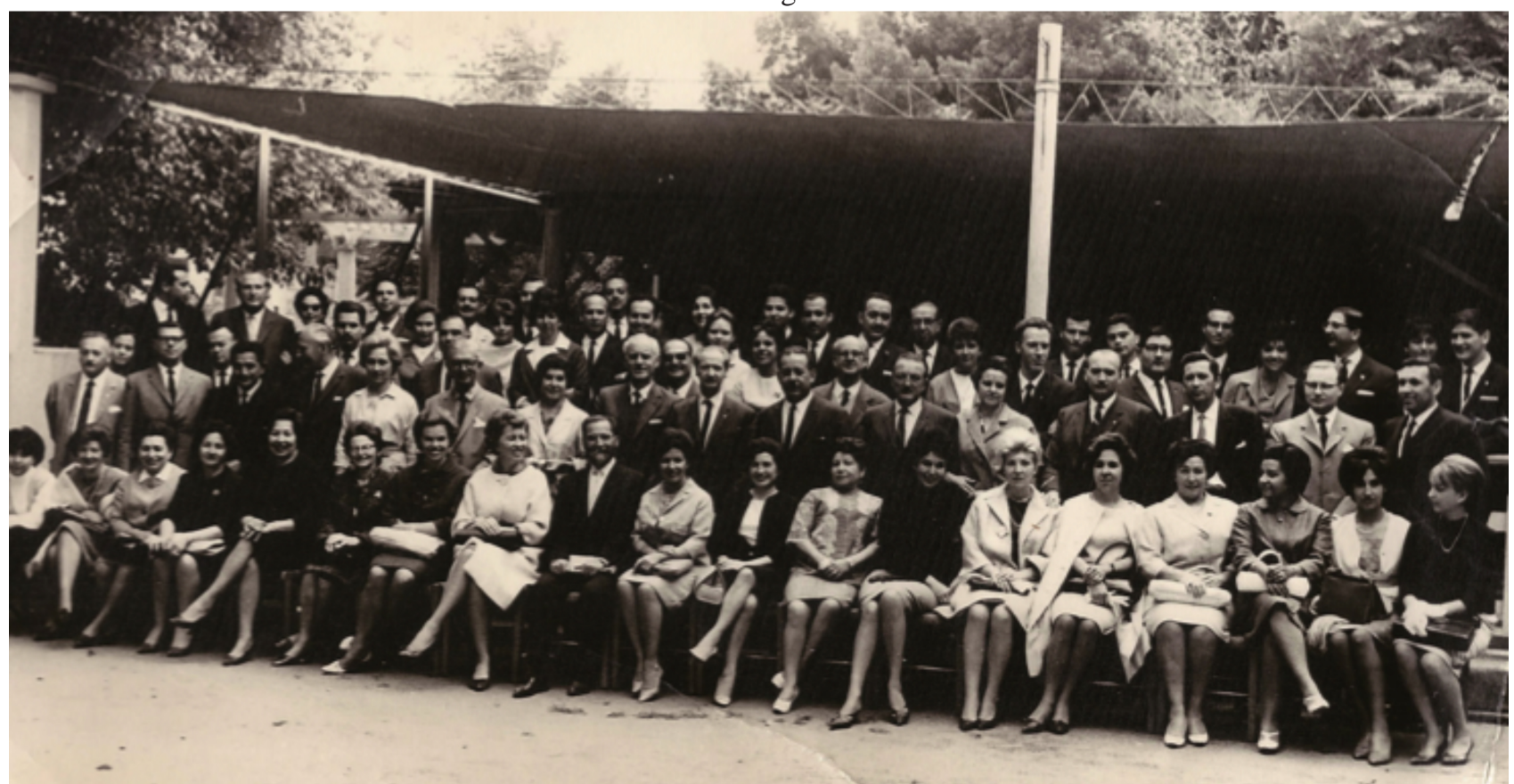

Fig. 5. Asistentes al almuerzo de clausura del $\mathrm{X}^{\circ}$ Congreso en Las Salinas - Viña del Mar, gentileza archivo personal Alberto Rodríguez. año 1965. 


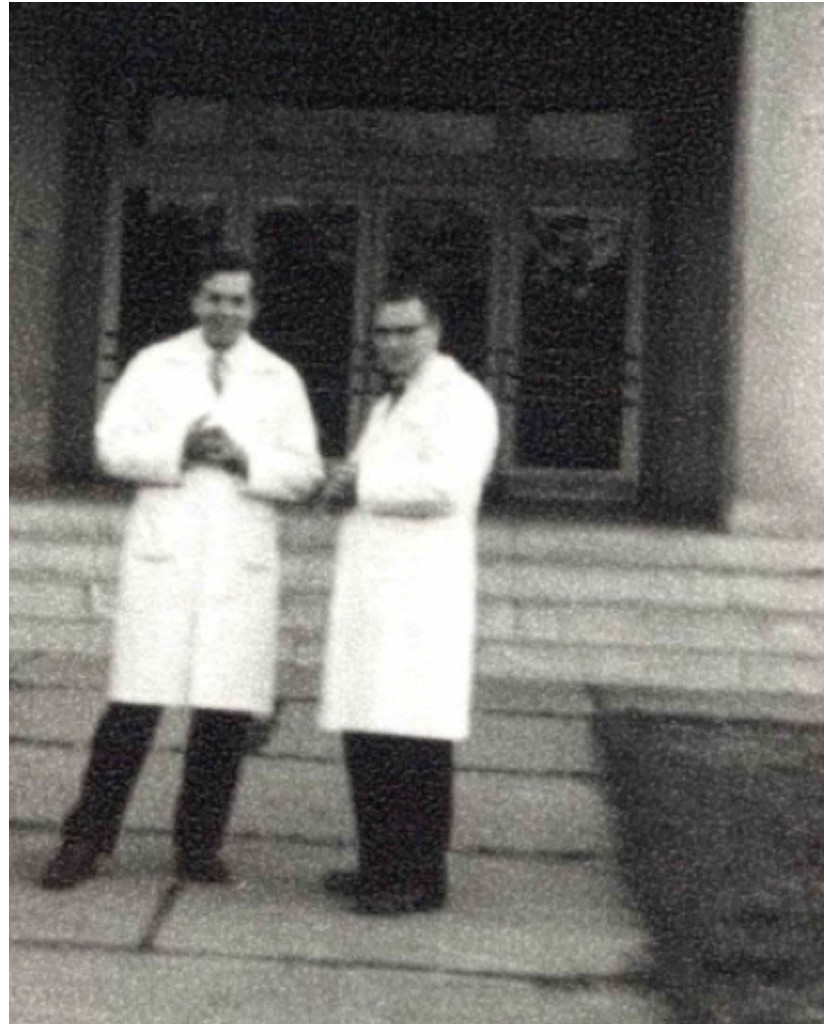

Fig. 6. Profesores Doctores Humberto Guiraldes del Canto, Alberto Rodríguez Torres, en el frontis del Instituto de Anatomía de la Universidad de Chile, año 1964. Gentileza del Prof. Dr. A. Rodríguez Torres.

Ya casi al finalizar el año 1970, se acrecienta esta separación interna cuando el Presidente de la Sociedad de esa época, Dr. Guillermo Rojas, de especialidad Anatomopatólogo, invita al Congreso de la Sociedad al recién asumido Presidente de la República Dr. Salvador Allende G., quien al inicio de su Carrera había tenido un breve desempeño como patólogo. Este hecho, en franca oposición a la mayoría de los miembros, se constituyó en el último acto de esta Sociedad mancomunada entre anatomistas y patólogos.

Como Presidentes de la Sociedad de Anatomía Normal y Patológica se recuerda a los profesores Gustavo Jirón L. fundador y primer presidente en el período 1938 - 1942. Le siguieron en el cargo de presidente hasta 1954 los siguientes (Tabla I).

\begin{tabular}{ll}
\hline Presidentes & Años \\
\hline Héctor Rodríguez R. & $(1943-1946)$ \\
Adolfo Escobar P. & $(1947-1948)$ \\
Roberto Barahona S. & $(1949-1950)$ \\
Miguel Ossandón G. & $(1951-1952)$ \\
Eduardo Calderón P. & $(1953-1954)$ \\
\hline
\end{tabular}

El último de los presidentes (1969 - 1970) sería el Dr. Guillermo Rojas Morales. (Actas de Directorio Soc. Anatomi Normal y Patológica)

Desde ese entonces, anatomistas y anatomopatólogos tomarían caminos distintos.

RODRÍGUEZ, T. A. \& CÁRDENAS, V. J. The origins of the society of normal and pathological anatomy of Chile. Int. J. Morphol., 35(2):751-755, 2017.

SUMMARY: From the beginnings, anatomists and pathologists are united in the year 1938 creating the Society of Normal and Pathological Anatomy Chilean in order to stimulate their investigations, seek to relate early with morphologists of the rest of America and to promote their publications in the Chilean Archives of Morphology. This is how Chilean morphologists participate for the first time in national and international congresses and conferences from the main universities in the country. Perhaps the work in common with the corpses was a fundamental point in its beginnings, more, the advance of the knowledge in each of these disciplines and later political differences would be two facts that would explain that they separated later in the year 1970. It would be therefore The first effort to gather around a Society of morphologists in Chile, would be the basis for the future Chilean Society of Anatomy.

KEY WORDS: Society of Normal and Pathologic Anatomy of Chile; History.

\section{REFERENCIAS BIBLIOGRÁFICAS}

Actas de Directorio Años 1938 a 1970. Santiago de Chile, Sociedad de Anatomía Normal y Patológica, 2017.

La Sociedad de Anatomia Normal y Patológica. Arch. Chil. Morfol., 3(4):9-10, 1940.

I Sesión de docencia y publicaciones. Arch. Chil. Morfol., 6:67, 1942.

Espinoza, A. \& Fuller, A. Anatomia Patológica, 132 Años de Historia, Huella y Presencia. Santiago de Chile, Facultad de Medicina Universidad de Chile, 2015.

Ferrer, P. L. Historia General de la Medicina en Chile. Talca, Imprenta Talca, 1904. pp.335.

Flores, N. Historia de la Anatomía en Chile. Arch. Chil. Morfol., 1(1), 1933.

Jirón, G. L. Los cuadros anatómicos clásicos y sus variaciones. Arch. Chil. Morfol., 6:47-51, 1946.

Sociedad Chilena de Anatomía. Sitio Web. Santiago de Chile, Sociedad Chilena de Anatomía, 2017. Disponible en: http://schap.cl/institucion/historia

Rodríguez, T. A. Entrevista. Santiago de Chile, Mayo 2014.

Rodríguez, T. A. Discurso de Agradecimiento del Profesor Dr. Alberto Rodríguez Torres, al Nombramiento como Maestro de la Anatomía de Chile. Int. J. Morphol., 23(1):43-4, 2005.

Solervicens, E. Los cuadros clásicos y sus variaciones. Arch. Chil. Morfol., 6:52-9, 1946.

Dirección para correspondencia:

Dr. Julio Cárdenas

Médico Cirujano Internista

Prof. Asistente ICBM, Anatomía y Biología del Desarrollo

Facultad de Medicina

Universidad de Chile

Santiago - CHILE

Recibido : 22-01-2017

Aceptado : 08-03-2017

Email: jcardena@med.uchile.cl 